\title{
INÉGALITÉS ÉDUCATIVES STRUCTURELLES AU BRÉSIL : ENTRE ÉTAT, PRIVATISATION ET DÉCENTRALISATION
}

\author{
Prof. Dr. Abdeljalil AKKARI*
}

\section{Résumé}

Dans cet article nous voulons soutenir la thèse que le système éducatif au Brèsil comprend non seulemente plusieurs réseaux, mais aussi des réseaux qui se développent à plusieurs vitesses, ce que ne permet pas d'établir une comparaison valable avec le modèle occidental.

Un regard sur l'histoire de l'éducation au Brèsil pousse le chercheur à percevoir que les conditions historiques de la constitution du système éducatif brèsilien ont engendré des réseaux opposés entre eux, sous la direction de l'Etat tout puissant.

Mots clés: réseaux, éducation, histoire, privé, public.

\section{Resumo}

Nesse texto queremos defender a tese de que o sistema educacional no Brasil é constituído não somente de diversas redes, mas, ainda, que essas redes se desenvolvem obedecendo a velocidades diferentes, fato que não permite estabelecer uma comparação válida com o modelo educacional ocidental.

Ao lançar um olhar sobre a história da educação no Brasil, o pesquisador é instado a perceber que as condições históricas da constituição do sistema educacional brasileiro deram origem a redes educacionais opostas, sob a direção do Estado todo-poderoso.

Palavras-chave: redes, educação, história, privado, público.

Université de Fribourg

Département des Sciences de l'éducation 


\section{Introduction}

La comparaison entre différents systèmes éducatifs a amené beaucoup de spécialistes à postuler l'existence d'un modèle universel d'éducation. Modèle vécu par les pays occidentaux dans la seconde moitié du 19 ème siècle et qui semble s'appliquer à d'autres régions du globe. Les système éducatifs des pays en voie de développement peuvent alors être assimilés à une " photocopie " décalé dans le temps et d'une qualité réduite par rapport au modèle occidental originel.

Dans ce texte, nous voulons atténuer cette thèse en postulant la constitution progressive au Brésil d'un système éducatif comprenant plusieurs réseaux à plusieurs vitesses. Ce système est difficilement comparable au modèle occidental. Le cas brésilien nous semble intéressant à plusieurs titres. Tout d'abord, le Brésil est le plus grand pays d'Amérique Latine par sa population et son territoire (et son développement économique). Ensuite, l'importance des travaux brésiliens réalisés sur ce thème nous donne un accès direct aux données et analyses locales. Enfin, des séjours réguliers durant les cinq dernières années sur le terrain nous ont permis de vérifier la lecture particulière que nous tentons de développer de la situation éducative brésilienne.

\section{Constitution historique des réseaux}

Dés le début du siècle, le développement du système éducatif brésilien est marqué par des rapports conflictuels entre différents groupes sociaux. Dans une recherche historique sur la pénétration protestante nord-américaine au Brésil, Mesquida (1994) explique que les élites intellectuelles et politiques de la région du Sud-est (Franc-maçons, républicains, anticléricaux, positivistes) n’ont pas hésité à soutenir l'église méthodiste dans sa tentative d'installer un enseignement alternatif à celui procuré par les écoles catholiques et publiques considérées comme passéistes, rigides et peu propice à impulser le développement économique.

Nous pouvons distinguer quatre périodes principales dans l'histoire des luttes en faveur de l'école publique au Brésil. La première (1934-1960) est marqué dans les années 30 par la discussion entre catholiques et laïcs sur les orientations générales de la politique éducative du pays (Libaneo, 1985)(dans le cadre de l'école nouvelle issue des Etats Unis, centrée sur Dewey). Entre 1956 et 1960, le débat s'est articulé autour du conflit entre école publique et école privée qui s'est développé. Elle a culminé avec la promulgation par le congrès d'une législation complète sur l'éducation (Lei de Diretrizes e bases).

La législation n'a pas mis fin au débat entre les défenseurs de l'école privée et ceux de l'école publique. Les premiers regroupés autour de l'église catholique défendaient une conception religieuse et humaniste de l'enseignement. Ils réclamaient même un financement public pour l'éducation privée afin de garantir la " liberté de choix " aux parents. Les seconds regroupés 
autour des mouvements progressistes et laïcs estimaient que seule l'école publique serait apte à garantir les mêmes chances éducatives pour tous les citoyens.

Sur le plan pédagogique, cette première période correspond à l'introduction de la pensée pédagogique libérale au Brésil avec notamment l'engagement des pédagogues libéraux en faveur d'une meilleure réponse à la demande sociale croissante d'éducation. Ce mouvement a culminé avec le lancement en 1932 du manifeste des pionniers de l'école nouvelle qui préconisait une universalisation de l'enseignement par le développement d'un système public d'éducation. Ce document considérait, l'enseignement comme une fonction sociale et publique (Azevedo et al., 1932)

Le renforcement de l'école publique sur le plan législatif après cette première période n'a pas provoqué une avancée massive dans la construction du système public. Les communautés défavorisées et les populations rurales restent à l'écart de la scolarisation massive.

La deuxième période correspond à l'apparition du mouvement d'éducation populaire qui s'est développé entre 1960 et 1964 grâce notamment au travail pionnier du mouvement d'éducation de base(MEB) et l'action du pédagogue Paulo Freire. Le débat s'est déplacé du champ scolaire vers celui de l'alphabétisation des adultes dans un contexte communautaire marqué par de multiples luttes sociales.

La troisième période a coïncidé avec l'avènement du régime militaire qui a donné un coup d'arrêt brutal à l'espoir suscité dans le pays par les campagnes d'alphabétisation populaire. Ce régime a tenté d'implanter politique éducative techniciste centrée autour des concepts de rationalité, efficience et productivité. Cette orientation inspirée notamment par les accords entre le Ministère de l'éducation et l'agence américaine d'aide au développement (USAID) a été combattue par la plupart des éducateurs brésiliens qui n'hésitaient pas à dénoncer le caractère autoritaire du régime et de sa proposition pédagogique (Saviani, 1992).

La quatrième période a coïncidé avec le retour progressif de la démocratie au début des années 80. Le débat s'est engagé autour de la démocratisation de l'enseignement et de la permanence des enfants défavorisés à l'école. De multiples mesures législatives en faveur de l'école publique ont été votées.

Ce bref rappel des conditions historiques de la constitution du système éducatif brésilien montre les principaux débats idéologiques du pays. D'abord quasi-monopole des ordres ecclésiastiques, l'éducation formelle a été progressivement organisée par l'état impérial, puis républicain pour accompagner le développement économique et la modernisation. Toutefois, cet Etat n'a jamais voulu ou pu contrôler l'ensemble du processus de scolarisation de masse tout au long du 20 ème siècle. L'enseignement privé s'est progressivement constitué comme seul alternative pour les enfants de l'élite. 
Le réseau public souffre de nombreuses faiblesse qualitatives et quantitatives malgré une législation et un discours politique omniprésents. Le résultat actuel est un système éducatif fragmenté, organisé en réseaux disparates difficilement comparables. Dans la section suivante, nous tenterons de décrire ces multiples réseaux.

\section{Caractéristiques du système éducatif brésilien}

Du point de vue quantitatif le système éducatif brésilien a réalisé de multiples progrès durant les dernières décennies. Une augmentation des taux de scolarisation à tous les niveaux de l'enseignement et une baisse constante du taux d'analphabétisme. Ainsi, ce dernier a passé de 39,5\% en 1960 à $20.1 \%$ en 1991 (Guimaraes, 1998). Donc sur le plan quantitatif la situation brésilienne est comparable à celle qui celle qui prévaut dans d'autres pays en voie de développement même si le Brésil a tendance à avoir de meilleurs résultats sur le plan économique qu'éducatif.

Dans le classement international sur la précarité des systèmes éducatifs dans les pays en voie de développement, le Brésil occupe sur le plan éducatif 16 places plus bas que son classement selon le PIB (Watkins, 1999)

La constitution de 1824 a reconnu le droit de chaque citoyen à une éducation primaire. En 1930, un ministère de l'éducation a été crée. Ensuite la loi directrice de 1962 a institué trois réseaux publics d'écoles (fédérales, des Etats et municipales). La constitution de 1988 explicite la coexistence du réseau public et privé. Pour le réseau privé, une distinction a été établi entre institutions à but lucratif et celles sans but lucratif (écoles communautaires, philanthropiques et confessionnelles).

Il nous semble que la dualité enseignement public / enseignement privé est la grille de lecture approprié pour analyser le système éducatif actuel au Brésil. Si le réseau public accueille la majorité des élèves, le réseau privé est de meilleure qualité et exclusivement fréquentés par les classes dominantes. 


\section{Tableau I Les résea ux public, privé et communa utaire de l'enseignement au Brésil}

\begin{tabular}{|c|c|c|c|}
\hline $\begin{array}{l}\text { Réseau public } \\
\text { Ecoles Fédérales }\end{array}$ & $\begin{array}{l}\text { Origine } \\
\text { Ecoles d'application } \\
\text { pedagogique liées à } \\
\text { des uníversités ou } \\
\text { des entreprises } \\
\text { publiques } \\
\text { Écoles fédérales } \\
\text { techniques } \\
\text { supérieures }\end{array}$ & $\begin{array}{l}\text { Public } \\
\text { Eléves issus des } \\
\text { classes supérieures et } \\
\text { moyennes }\end{array}$ & $\begin{array}{l}\text { Caractéristiques } \\
\text { Très peu } \\
\text { nombreuses } \\
\text { avec peu de places } \\
\text { disponíbles. } \\
\text { Concours d'entrée } \\
\text { très sélectifs }\end{array}$ \\
\hline Ecoles des Etats & $\begin{array}{l}\text { Ecoles gérées par les } \\
\text { états régionaux }\end{array}$ & $\begin{array}{l}\text { Elèves issus des } \\
\text { classes moyennes et } \\
\text { défavorisés }\end{array}$ & $\begin{array}{l}\text { Assez nombreuses } \\
\text { mais leurs } \\
\text { nombres est en } \\
\text { diminution suite à } \\
\text { ia } \\
\text { municipalisation } \\
\text { de l'éducation }\end{array}$ \\
\hline Ecoles Municipales & $\begin{array}{l}\text { Ecoles gérées par les } \\
\text { municipalités }\end{array}$ & $\begin{array}{l}\text { Eléves issus des } \\
\text { classes défavorisées }\end{array}$ & $\begin{array}{l}\text { Les plus } \\
\text { nombreuses } \\
\text { Taux de } \\
\text { redoublement et } \\
\text { d'évasion très } \\
\text { importants }\end{array}$ \\
\hline \multicolumn{4}{|l|}{ Réseau privé } \\
\hline $\begin{array}{l}\text { Ecoles } \\
\text { internacionales }\end{array}$ & $\begin{array}{l}\text { Localisées } \\
\text { essentiellement à } \\
\text { São Paulo et Rio de } \\
\text { Janeiro }\end{array}$ & $\begin{array}{l}\text { Elèves issus des } \\
\text { classes supérieures } \\
\text { (professionnels } \\
\text { étrangers) }\end{array}$ & $\begin{array}{l}\text { Peu nombreuses } \\
\text { Elles sont réputées } \\
\text { dans le secteur de } \\
\text { l'éducation } \\
\text { biblingue } \\
\text { (essentiellement } \\
\text { portugais-Anglais) }\end{array}$ \\
\hline $\begin{array}{l}\text { Écoles } \\
\text { "traditionnelles" } \\
\text { confessionnelles }\end{array}$ & $\begin{array}{l}\text { Al:origine liées aux } \\
\text { des puissantes } \\
\text { congrégations }\end{array}$ & $\begin{array}{l}\text { Elèves issus des } \\
\text { classes supérieures et } \\
\text { des classes } \\
\text { moyennessupérieures }\end{array}$ & $\begin{array}{l}\text { Leur nombre est en } \\
\text { constante } \\
\text { augmentation }\end{array}$ \\
\hline
\end{tabular}




\begin{tabular}{|c|c|c|c|}
\hline $\begin{array}{l}\text { (catholiques, } \\
\text { protestantes }\end{array}$ & $\begin{array}{l}\text { catholiques (jesuites, } \\
\text { maristes, } \\
\text { dominicains), ou } \\
\text { protestantes } \\
\text { (méthodistes, } \\
\text { évangéliques, etc.), } \\
\text { ces écoles se sont } \\
\text { fortement } \\
\text { développées ces } \\
\text { dernières années }\end{array}$ & & $\begin{array}{l}\text { L'enseignement } \\
\text { religieux perd du } \\
\text { terrain à mesure } \\
\text { que leur base } \\
\text { confessionnelle de } \\
\text { recrutement } \\
\text { s'élargit }\end{array}$ \\
\hline Ecoles "entreprises" & $\begin{array}{l}\text { A l'origine des écoles } \\
\text { spécialisées dans la } \\
\text { préparation de } \\
\text { l'examen d'entrée à } \\
\text { l'université } \\
\text { (vestibulaire). Ce } \\
\text { sont de véritables } \\
\text { entreprises de } \\
\text { l'education á } \\
\text { l'échelle nationale. }\end{array}$ & $\begin{array}{l}\text { Eléves issus des } \\
\text { classes supérieures et } \\
\text { moyennes- } \\
\text { supérieures }\end{array}$ & $\begin{array}{l}\text { Leurs nombre est } \\
\text { en augmentation } \\
\text { exponentielle } \\
\text { Activités très } \\
\text { diversifiées } \\
\text { comprenant } \\
\text { notamment un } \\
\text { secteus d'édition } \\
\text { très dynamique et } \\
\text { même des unités de } \\
\text { production } \\
\text { d'ordinateus et de } \\
\text { logiciels éducatifs. } \\
\text { Un exemple } \\
\text { typique est celui du } \\
\text { groupe Positivo qui } \\
\text { regroupe } 500 \text { ooo } \\
\text { éleves (à vérifier') }\end{array}$ \\
\hline $\begin{array}{l}\text { Petites écoles } \\
\text { privées }\end{array}$ & $\begin{array}{l}\text { De création } \\
\text { relativement récente } \\
\text { (souvent des } \\
\text { entreprises } \\
\text { individuelles/ } \\
\text { familiales), ce sont } \\
\text { de petites écoles } \\
\text { maternelles ou } \\
\text { primaires qui se sont } \\
\text { développées sur des } \\
\text { initiatives } \\
\text { individuelles avec } \\
\text { peu de classes (une } \\
\text { maison individuelle) }\end{array}$ & $\begin{array}{l}\text { Elèves issus des } \\
\text { classes moyennes }\end{array}$ & $\begin{array}{l}\text { Flexibilité } \\
\text { Qualité est très } \\
\text { variable } \\
\text { Certaines peuvent } \\
\text { être } \\
\text { assimilées à des } \\
\text { écoles publiques } \\
\text { (enseignement } \\
\text { supplétif pour } \\
\text { adultes) }\end{array}$ \\
\hline
\end{tabular}




\begin{tabular}{|l|l|l|l|}
\hline $\begin{array}{l}\text { Réseau } \\
\text { communautaire }\end{array}$ & & \\
\hline $\begin{array}{l}\text { Ecoles } \\
\text { communautaires }\end{array}$ & $\begin{array}{l}\text { Des écoles crées par } \\
\text { des mouvements } \\
\text { communautaires ou } \\
\text { syndicaux pour } \\
\text { pallier l'absence de } \\
\text { d'une offre } \\
\text { d'éducation publi- } \\
\text { que }\end{array}$ & $\begin{array}{l}\text { communautes des } \\
\text { rurales isolées }\end{array}$ & $\begin{array}{l}\text { Ecoles autogérées et } \\
\text { largement auto } \\
\text { financées par les } \\
\text { communautés } \\
\text { locales }\end{array}$ \\
& & \\
\hline
\end{tabular}

Le tableaux I montre l'existence d'au moins trois réseaux d'enseignement primaire et secondaire représentés par différents types d'école. Les services éducatifs (quantité et qualité) de ces réseaux sont très variables à l'intérieur d'un même Etat, mais aussi entre Etats. En particulier, dans les régions rurales et excentrées, l'offre qui se limite souvent à des écoles municipales et des états. L'enseignement privé y est très peu développé. Les classes favorisées de ces régions n'hésitent pas à scolariser leurs enfants à des dizaines de kilomètres de leur domicile.

Les disparités entre Etats sont également flagrantes. Le Nord-Est en particulier dispose d'un réseau public très dégradé. L'examen du nombre d'enseignants leigos (sans formation pédagogique) est l'un des indicateurs qui permet de constater la précarité de l'éducation publique.

\section{Tableau II Professeurs Leigos à l'école primaire au Nord et au Nordest}

\begin{tabular}{|l|c|l|l|}
\hline Etats & $\begin{array}{l}\text { Nombre de } \\
\text { professeurs }\end{array}$ & $\begin{array}{l}\text { \% des professeurs } \\
\text { avec le primaire } \\
\text { incomplet }\end{array}$ & $\begin{array}{l}\text { \% de professeur } \\
\text { avec } \\
\text { l'enseignement } \\
\text { moyen incomplet }\end{array}$ \\
\hline Rondonia & 8.104 & 12.1 & 31 \\
Pará & 36.365 & 11.7 & 29.5 \\
Tocantins & 8.463 & 17.2 & 31.1 \\
Maranhão & 39.909 & 18.7 & 29.7 \\
Piauiu & 22.773 & 16.6 & 27.1 \\
Ceará & 44.048 & 10.8 & 24.5 \\
Bahia & 77.278 & 10.1 & 19.9 \\
Sud-Est & 267.718 & 0.6 & 1.1 \\
Sud & 113.162 & 0.9 & 4.2 \\
Brèsil & 798.947 & 5.5 & 1.9 \\
\hline
\end{tabular}


Le Nord-Est brésilien constitue la plus grande concentration de pauvreté en Amérique Latine. Plus de la moitié des enfants dans les zones rurales du Nord-Est reçoivent moins de 4 ans de scolarité et un quart de la population n'a pas été scolarisée (Watkins, 1999)

Il est également important de noter que les réseaux sont "dynamiques" et non pas "statiques". La qualité du réseau public dépend de la politique éducative menée sur le plan municipal, étatique et fédéral. Il est par exemple indéniable que le réseau de la ville de Sao Paulo s'est amélioré sous l'administration de Paulo Freire(Je pense que la contribution de Freire pour le développement du systhème éducatif paulistano ne soit pas être survalolrisé, cela parce que : 1. Freire n'a jamais cru à l'éducation formelle ;2. Il a passé trop peu du temps à la tête du sécretariat municipal d'éducation pour exercer une influence durable sur la structure du système).

La situation du réseau privé dépend des incitations fiscales de l'Etat et du degré de contrôle dont il est soumis. La constitution de 1988 a théoriquement limité le transfert des ressources publiques vers l'enseignement privé. Mais, les transferts indirect continuent notamment à travers l'achat massif de manuels scolaires chez des éditeurs privés (Akkari, In Press).

Il existe également une circulation possible des élèves entre les différents réseaux. Un même élève peut sortir d'un réseau pour entrer dans un autre. Mais, il ne s'agit pas d'un "marché concurrentiel" puisque chaque réseau a son public.

Les stratégies familiales sont à cet égard très multiples, Les familles de la classe moyenne supérieure maintiennent parfois leurs enfants dans le public pour le primaire mais les transfèrent dans les réseaux privés pour le secondaire en raison notamment de la perspective de l'examen d'entrée à l'université [Vestibular]. De même, certaines familles de la classe moyenne peuvent transférer leurs enfants des petites écoles privées vers les écoles publiques quand les frais de scolarité augmentent fortement ou que leurs situations économiques se dégradent.

Toutefois, il faut souligner que la mobilité entre les réseaux n'est pas possible pour tous et qu'elle est étroitement liée au pouvoir d'achat. Les classes populaires n'ont qu'un seul choix: celui des écoles municipales pas toujours existantes dans les zones rurales. Les enfants sont parfois obligées de sortir du système éducatif pour s'insérer rapidement dans le tissu productif et contribuer à la survie de la famille. En 1990, 11 \% des enfants de 10 à 14 ans travaillent au Brésil (32\% en milieu rural) (Saboia, 1996).

Les résultats d'une récente enquête nationale montrent clairement la consolidation d'un système éducatif brésilien à plusieurs réseaux et plusieurs vitesses. Les disparités entre régions en matière d'acquisitions des élèves sont indéniables. 


\section{Tableau III}

Score moyen en mathématiques des élèves de 4 ème année primaire par région

\begin{tabular}{|l|l|l|}
\hline & Urbain & Rural \\
Brésil & 189 & 167 \\
Nord & 169 & - \\
Nord-Est & 178 & 161 \\
Sud-Est & 196 & 171 \\
Sud & 195 & 181 \\
Centre-Ouest & 186 & 165 \\
\hline
\end{tabular}

Source: Gomes de Sá Pestana et al. (1999)

\section{Tableau IV}

Score moyen en mathématiques des élèves de 4 ème année primaire par type d'école

\begin{tabular}{|l|l|l|l|}
\hline & Ecoles étatiques & Ecoles municipales & Ecoles privées \\
Brésil & 183 & 177 & 230 \\
Nord & 167 & 163 & 213 \\
Nord-Est & 170 & 164 & 220 \\
Sud-Est & 191 & 184 & 238 \\
Sud & 192 & 188 & 236 \\
Centre-Ouest & 180 & 177 & 231 \\
& & & \\
\hline
\end{tabular}

Source: Gomes de Sá Pestana et al. (1999)

Les salaires des enseignants est un autre indice de la disparité entre réseaux. 
Salaire moyen des enseignants du primaire (1 à 4) par région et dépenda nce administrative (en $R \$)$

\begin{tabular}{|llllll|}
\hline & $\begin{array}{l}\text { Ensemble } \\
\text { des écoles }\end{array}$ & $\begin{array}{l}\text { Ecoles } \\
\text { fédérales }\end{array}$ & $\begin{array}{l}\text { Ecoles } \\
\text { étatiques }\end{array}$ & $\begin{array}{l}\text { Ecoles } \\
\text { municipales }\end{array}$ & $\begin{array}{l}\text { Ecoles } \\
\text { privées }\end{array}$ \\
Brésil & 420,10 & 1103,3 & 515,5 & 292,7 & 595,30 \\
Nord & 365,8 & 1296,4 & 456,70 & 229,0 & 529,2 \\
Nord-Est & 222,00 & 438,10 & 344,70 & 155,2 & 288,10 \\
Sud-est & 617,7 & 1346,5 & 616,20 & 545,5 & 782,7 \\
Sud & 464,20 & 978,20 & 499,40 & 405,80 & 681,20 \\
Centre-ouest & 456,40 & 1135,90 & 451,4 & 315,3 & 562,5 \\
\hline
\end{tabular}

Source: Guimaraes (1998)(Pourquoi ne pas dollariser les chiffres ? 1 US\$= 2 R $\$$ )

Le coût par élève est également un indice de la différence de qualité entre les réseaux public et privé, comme nous mostrerons dans la suite de ce texte.

En 1997, Da Silva (1998) relève que le coût mensuel moyen d'un mineur interné dans une institution de la "Febem" est de 1.177 R\$, soit 24 fois le montant par élève scolarisé dans le réseau étatique de Sao Paulo. (Je te rappelle que la FEBEM ne s'occupe que des enfants Qui ont commis de crimes tels que vols, homicides, latrocides, et.)

Il est intéressant de noter que la structuration du primaire et du secondaire en réseaux détermine le recrutement de l'enseignement supérieur. En effet, en raison de la difficulté extrême d'examen d'entrée dans les universités publiques, il n'est pratiquement pas possible de trouver des étudiants ayant fréquenté l'enseignement public dans le primaire et le secondaire dans les universités publiques. Ces dernières recrutent (presque) exclusivement ( exclusivement pour les sections les plus réputés) des élèves issus du secondaire privé. Curieusement quand tous les élèves pauvres ont été éliminés, l'enseignement devient "public" et "gratuit". Le Ministère Fédéral de l'éducation dépense $60 \%$ de ses ressources sur les universités alors que $20 \%$ des professeurs de l'enseignement fondamental (éducation de base) vivent avec moins de deux salaires minimums : R\$200 (Tarumann, 1999)

Dans les cours les plus demandés dans les universités publiques sont fréquentés par 80 \% d'élèves qui ont passé par l'école privée. Ce pourcentage descend à $40 \%$ pour les disciplines les moins demandées. Or, les élèves qui terminent l'enseignement moyen public sont trois plus nombreux que ceux qui terminent l'enseignement secondaire privé. Dans le concours d'accès aux 
facultés publiques de médecine, 1 élève provenant du privé a une chance sur 9 pour réussir et celui qui provient du public une chance sur 104 (Lima, 1999).

(Il ya un projet de loiqui a été voté au parlement et attende la sanction du présidente de république déterminant que la moitié des places dans les isntitutions fédérales d'enseignement supérieur soit reservées aus étudiants pauvres, donc issus de l'école sécondaire publique) L'enseignement privé universitaire recrute une partie de sa clientèle de l'enseignement public secondaire (à développer).

Conséquence de la ségrégation résidentielle: une socialisation à plusieurs vitesses.

Pourquoi le modèle éducatif brésilien diverge du modèle occidental ?

Le modèle occidental se caractérise par :

- un consensus sur le caractère éminemment public de l'éducation de base

- l'existence de l'enseignement privé est souvent lié à des considérations religieuses et régionales (la Bretagne en France)

- Il existe souvent des accords (des contrats) entre l'enseignement privé et l'état (par exemple la prise en charge des salaires par l'Etat) et un contrôle pédagogique important

- La différence en matière de dépenses par élève entre public et privé est peu importante

- La reproduction des élites n'est pas exclusivement du ressort de l'éducation de base privée

Signalons tout de même certains auteurs estime que le modèle éducatif occidental est également sur la voie d'une fragmentation croissante, cachée en Suisse (Hutmacher, 1900) ou beaucoup plus flagrante en France et aux USA (Kozol, 1900, Careil, 1998)

\section{Les piliers du système à réseaux Néolibéra lisme et privatisation}

Au Brésil, les années 90 sont marquées par un climat de perplexité et de désarroi général à propos de l'éducation. Le gouvernement Cardoso à orientation néo-libérale se caractérise par une politique éducative incohérente combinant un " discours sur l'importance de l'éducation " et un " désengagement de l'Etat" du secteur avec le rôle croissant des ONG et de l'initiative privée (Saviani, 1996)

Comme le signale Frigotto (1996), la thèse centrale du néolibéralisme est que le secteur public (l'Etat) est responsable de la crise, des privilèges et de l'inefficience. Le marché et le secteur privé sont synonymes 
d'efficience, de qualité et d'équité. La solution devient alors l'Etat minimal et la nécessité de mettre en question toutes les conquêtes sociales comme la stabilité de l'emploi, le droit à la santé, à l'éducation et aux transports publics.

L'Etat doit être réduit à la proportion congrue juste nécessaire pour la reproduction du capital. Sur le plan éducatif le néo-libéralisme se traduit par l'idée centrale du marché comme mécanisme de régulation. L’Etat devrait fournir à chaque famille une somme lui permettant d'acheter sur le marché libre, le service éducatif qui lui convient. Par ailleurs, de multiples entreprises privées parrainent ("adoptent") des écoles publiques. La philanthropie du partenariat se trouve ainsi élevé au rang d'une politique éducative de l'Etat (Frigotto, 1996).

Frigotto (1996) signale que l'apparition de multiples ONG, qui se disputent les fonds publics et privés a un double effet pervers. Ces ONG de nouveau type (à but exclusivement lucratif) compromettent le travail ancien des ONG traditionnelles effectivement engagées socialement. De plus, cela donne l'illusion que les ONG constituent une alternative démocratique locale à la démission de l'Etat et à son désengagement social.

\section{Critique de l'éducation comme marché}

Les consommateurs de l'éducation, considérée comme marché peuvent découvrir quand il est trop tard que la main invisible du marché ne peut pas être blâmée pour les défauts et les échecs de la privatisation simplement parce qu'elle ne peut être localisée. Les espaces publics et démocratiques régulateurs qui peuvent la dénoncer n'existent pas et ne peuvent être mis en ouvre faute de mobilisation populaire suffisante. Quand les concepts de qualité (de manque de qualité), voire de qualité totale remplacent les concepts d'inégalité structurelle et d'injustice dans le débat, la reproduction des inégalités sociales par un système éducatif à plusieurs réseaux se trouve largement facilitée.

Concernant le rôle de l'école dans la reproduction des inégalités sociales, nous pouvons distinguer à partir de 1934, trois moments dans l'histoire scolaire brésilienne. Le premier moment correspond à la période historique où la reproduction sociale se passe largement de l'école, ou l' "élu " peut hériter son capital industriel ou agricole sans titre scolaire. Les stratégies déployées sont alors centrées sur la famille ou les alliances matrimoniales. Le contrôle de la production du café et du lait assurait l'hégémonie des élites du Sud-est sur le pays. Le capital scolaire comme stratégie supplémentaire de renforcement du pouvoir des classes dominantes était secondaire. Le véritable acteur du jeu scolaire durant ce premier moment est l'Etat qui était préoccupé dans les années 40-50 et 60 de recruter à travers la scolarisation de masse sa base administrative et une main-d'œuvre qualifiée pour son modèle de développement basé sur la substitution de l'importation. Le lancement compagne national d'éducation rurale du gouvernement Getulio Vargas visait 
l'élévation du niveau économique des régions rurales à travers l'emploi des techniques modernes de production (Barreiro, 1900).

Le deuxième moment correspond à la dictature militaire (1964-1985) qui peut être considéré comme une période où le débat éducatif était réduit à un agencement techniciste. C'est le triomphe de la pédagogie comme technique de transmission de connaissances neutre socialement et politiquement. L'institution d'une contribution appelée salaire-éducation dans les entreprises de plus de 100 employés durant le régime militaire, permettait l'achat des places dans l'enseignement privé (Zibas, 1997). Ce deuxième moment nous semble extrêmement important dans la mesure ou il a mis fin à la lutte des classes populaires et il a préparé le troisième moment (néo-libérale) en dépolitisant le débat éducatif.

Le troisième moment commence au début des années 80 marque un retour du débat démocratique sur l'éducation après de gel des esprits durant la période militaire. Le capital scolaire occupait un rôle de plus en plus important. Les familles et les groupes sociaux dominants mettent alors en ouvre des stratégies calculées pour s'en assurer la maîtrise du capital scolaire notamment à travers la privatisation et la décentralisation de l'enseignement.

Le démantèlement de l'éducation publique et la segmentarisation de l'enseignement privé deviennent ainsi des outils prépondérants. Comme par ailleurs, la maîtrise du capital non-scolaire est quasi-définitivement assurée pour certaines familles (Cf. chiffres sur la répartition de la rente brésilienne Folha de Sa Paulo), le jeu devient alors principalement scolaire notamment pour les membres des classes moyennes supérieures. Contrôler la mobilité scolaire devient l'une la seule possibilité pour espérer une mobilité sociale.

Comme le souligne Zibas (1997) les arguments éthiques et philosophiques anciens au Brésil sur les prétendus avantages de l'école privée (droit de la famille, pluralité idéologique et religieuse) se sont transformés en une rationalité de type administrative et économique qui même sans base empirique solide défend la qualité de l'enseignement privé.

\section{Décentra lisation et municipalisation}

La progressive décentralisation de l'enseignement fondamental rendu possible par le retour à la démocratie n'a pas été accompagnée de mesure d'appuis financier et technique suffisants, ni d'une garantie quant à la qualité de l'enseignement dispensé dans les écoles municipales.

Le processus de décentralisation de l'éducation s'est constitué au Brésil comme un mécanisme vertical (du haut vers le bas) et antidémocratique. L'Etat Fédéral va progressivement déléguer aux Etats régionaux, aux municipalités aux ONGs et aux communautés locales la gestion de l'éducation publique de base sans pour autant mettre en place une réforme fiscale qui permet d'assurer un financement adéquat dans toutes les régions du pays. Le gouvernement 
central semble également ignorer la tradition clientéliste et le physiologisme qui caractérisent la classe politique de l'intérieur fortement contrôlée par les



Même dans un contexte d'un pays développé comme la France, certains spécialistes s'élèvent pour dénoncer les conséquences de la décentralisation sur l'aggravation des inégalités éducatives: "Dés lors que les sommes traditionnellement versés par les municipalités s'avèrent de moins en moins suffisantes pour faire fonctionner au quotidien les écoles que l'on voudrait tout à la fois "ouvertes" et bien équipées, les instituteurs se voient contraints, plus encore que par le passé, de recourir à des formes nouvelles de financement et font de plus en plus appel aux parents " (Careil, 1998). La richesse des activités pédagogiques offertes aux élèves devient encore plus directement liée aux revenus de leurs parents.

Selon Watkins (1999), le Brésil possède actuellement un système de financement de l'éducation de base le plus décentralisé de la région latinoaméricaine sans progrès notable en matière d'éducation publique. 33 Etats et plus de 45000 municipalités partagent avec le gouvernement fédéral la responsabilité du financement de l'éducation de base. Dans les zones rurales du Nord-Est, la moyenne annuelle des dépenses par élève des écoles municipales est de R $\$ 50$ comparées alors que les dépenses dans les écoles dépendant des Etats (situées principalement en zone urbaine) s'élèvent à 300 $\mathrm{R} \$$. La disparité entre les écoles municipales rurales du Nord-Est et les écoles des Etats du Sud-Est est encore plus grande puisque les dépenses par élèves sont 20 fois supérieures dans les secondes

L'une des raisons de ces énormes disparités en matière de financement de l'éducation de base au Brésil provient du fait que la décentralisation renforce l'inégalité entre Etats, communautés et régions. Les Etats régionaux récoltent la moitié des impôts au Brésil principalement grâce aux impôts sur les ventes. Logiquement, les régions et les Etats économiquement développés de la fédération récoltent les revenus les plus importants, Les revenus sont ainsi inversement proportionnels aux besoins de la population. Les dépenses municipales sont principalement assurées par des transferts fédéraux et certaines taxes locales. Même s'il existe un mécanisme de solidarité fiscale, il demeure encore embryonnaire. De plus, la pression exercée par les Fonds monétaire international sur les finances fédérales contribue à précariser le financement de l'éducation. Ainsi, Watkins (1999) rapporte qu'en 1996, un Fonds de stabilisation fiscal a été créé pour réduire le déficit national. Près d'un cinquième des revenus du gouvernement sont actuellement automatiquement transférés sur ce fonds. Une partie de cet "ajustement" est supporté par le budget fédéral d'éducation, ce qui a réduit le transfert aux municipalités de $18 \%$.

Le pacte des élites

Même dans les pays où la vague néo-libérale était triomphante comme 
les USA de Reagan et la Grande Bretagne de Thatcher, le système éducatif public a relativement bien résisté grâce notamment à la mobilisation syndicale et l'engagement traditionnel d'une partie de la classe moyenne en faveur de l'école publique.

Au Brésil, les catégories qui peuvent contester la privatisation rampante de l'enseignement comme les enseignants des écoles publiques, les syndicalistes et les élites progressistes sont eux même immobilisés par l'incohérence de leur inclinaison à mettre leurs enfants dans l'enseignement privé quand ils prétendent défendre l'enseignement public.

L'autre difficulté perverse est reliée du débat empoisonné amené par la Banque mondiale sur la prétendue nécessité absolue de réorienter les fonds publics de l'enseignement supérieur vers l'éducation de base. Alors que les universités publiques sont peut-être le dernier lieu ou une défense sincère de l'éducation publique est entreprise. (Les universités publiques sont les isntitutions Qui peuvent aider le pays à s'en sortir de la dépendance en technologie au travers de la recherche scientifique, simplement détruite par le gouvernement FHC)

Dans le même temps, certains auteurs brésiliens que l'on ne peut pas taxer de conservateurs estiment curieusement que le débat n'est plus à poser en terme de dualité entre l'enseignement public et privé. Ainsi, Gadotti (1992) ancien collaborateur de Paulo Freire, estime curieusement qu'il est erroné d'opposer l'enseignement public et privé. Il pense que toutes les familles idéologiques se trouvent au Brésil dans la défense de l'un ou de l'autre.

Il est vrai que l'église catholique par exemple, même si elle dirige un réseau privé à clientèle élitiste n'hésite pas à financer des écoles communautaires pour les élèves défavorisés. Mais, il est tout de même flagrant que l'enseignement privé ne profite qu'une minorité de la population.

Dans un article récent intitulé "enseignement privé ou public : Est une fausse question ", De Moura Castro (1997) illustre la collusion de certains spécialistes de l'éducation avec les orientations néo-libérales du gouvernement actuel. Ainsi, cet auteur se félicite que les familles de meilleures conditions économiques retirent leurs enfants en fin du primaire public pour les mettre dans l'enseignement privé car cette démarche augmente leur " compétitivité " dans l'examen d'entrée à l'université [vestibular]. L'auteur affirme que l'enseignement privé est $50 \%$ plus efficient à amener les élèves à la fin du cycle secondaire. Or, non seulement les deux réseaux (public et privé) diffèrent sur le plan du coût par élève (par exemple nombre d'élève par classe Cf. IBGE), mais aussi sur le plan de l'origine sociale de leur public respectif.

L'auteur prétend que l'enseignement privé a une meilleure efficience dans l'utilisation des ressources disponibles. C'est tout à fait probable, mais il 
faut tout de même rappeler que les frais mensuelles de scolarité par élève dans une école privée dépassent souvent les dépenses annuelles par élève dans les écoles publiques (sans même tenir compte des dépenses annexes des familles favorisées ; clubs, cours particuliers, équipement en logiciels éducatifs etc..).

L'inefficience du système public comparé au privé est présentée sans aucune référence à ses ressources disponibles. De plus, les ressources nécessaires pour augmenter la fréquentation de l'enseignement privé ne peuvent résulter d'un transfert des fonds publics tout simplement parce que ces fonds publics n'existent pas en quantité suffisante.

Le système des vouchers encensé par la littérature néo-libérale n'a fait ses preuves dans aucun pays du monde.

Ensuite, De Moura Castro (1997) prétend que l'enseignement privé occupe les espaces laissés par l'Etat “ Quand l'Etat n'arrive pas la périphérie, le privé répond en ouvrant des écoles. Quand la qualité de l'enseignement public laisse à désirer, le privé offre des écoles de meilleure qualité. Quand, il n'y pas de places dans l'enseignement public, le privé se charge de les procurer ". A en croire De Moura Castro, l'entrepreneur privé de l'éducation est un véritable philanthrope!.

En réalité, l'auteur se contredit avec le début de son article quant il a rapporté les chiffres du Ministère de l'éducation qui montrent que les régions économiquement les plus développées du Brésil comportent la plus forte proportion d'élèves dans l'enseignement privé.

Pour couronner son apologie pour l'enseignement privé, l'auteur estime qu'il comporte plus de "diversité ", qu'il peut être auto-piloter, économe en matière d'énergie administrative et de pouvoir public! (" autigovenado, economizando energias administrativas, do poder publico). Fonctionnaire de la Banque Interaméricaine de Développement, l'auteur semble confondre une analyse du système éducatif brésilien avec les orientations idéologique de son institution d'affectation.

La banque Mondiale recommande clairement au Brésil une politique de privatisation de l'enseignement. Mais cette politique devrait selon la Banque Mondiale inclure un financement public pour les entrepreneurs privés de l'éducation afin qu'ils construisent des écoles dans les zones défavorisées (World Bank, 1989)

Pour conclure cette partie, il nous semble important de signaler qu'il est très difficile de rompre la logique des réseaux dans le climat actuel de démobilisation par rapport à l'enseignement public.

Sans doute, on ne mesurera jamais assez les conséquences de l'instauration du régime militaire sur la démobilisation des classes moyenne et populaire par rapport à la défense de l'école publique (à développer) 


\section{Alterna tives possibles}

Dans le domaine de la politique éducative, nous pouvons observer deux orientations, la première a une confiance absolue dans le marché comme agent de régulation sociale et une autre orientation qui considère le rôle de central l'Etat dans la lutte contre les inégalités (Zibas, 1997).

Soulignant tout d'abord les difficultés de lutter contre le néo-libéralisme et le rafistolage de façade.

Curry \& Nogueira (1989) rappellent que l'école est aussi un produit de luttes sociales. Dans ce sens le réseau public d'enseignement aura probablement une autre configuration à l'intérieur d'un nouvel ordre politique et social. Les ressources publiques pour le réseau public signifient une " républication " (republicação) d'un Etat qui en se privatisant a laissé l'espace ouvert aux entrepreneurs de l'éducation pour qu'ils occupent la " face publique " de l'Etat.

Parallèlement à la critique de la mercantilisation de l'enseignement, il faut dénoncer la privatisation de l'Etat qui a permis cette mercantilisation. Audelà de la critique, Curry \& Nogueira (1989) appellent à une politique éducative ou l'Etat ne sera plus un lieu du pouvoir des groupes hégémoniques de la société. La réorientation de la politique éducative de l'Etat sera également l'indice qui permet de mesurer la participation des groupes subalternes à la citoyenneté

En analysant le plan décanal d'éducation pour tous (1993-2003), Carvalho Mazzeu (1995) estime que l'ancrage pédagogique du plan demeure techniciste avec les caractéristiques suivantes :

- accent sur la productivité et l'efficience de l'école

- priorité à l'établissement d'objectifs éducatifs nationaux en termes de compétences (habilités) qui doivent être maîtrisées par les élèves et les enseignants.

- Accent sur les ressources matérielles plus particulièrement sur les techniques d'éducation à distance comme une solution à la baisse de "qualité de l'enseignement" et comme une option pour la formation des professeurs (équation coûts-bénéfices)

- Absence d'une discussion de fond sur la fonction politique de l'école ou son rôle dans la formation du citoyen.

En arrière plan, on perçoit le mythe d'une société homogène et d'une neutralité politique de l'éducation. Aucune volonté d'offrir une éducation de niveau comparable dans les réseaux public et privé

A partir de 1986, le Gouvernement a lancé divers programmes nationaux d'alimentation scolaire (PNAE), du livre scolaire (PNLD) et du matériel scolaire (PNME).

Le PNLD a enregistré entre 1984 et 1985 un accroissement de $74 \%$. En 1987, 55 millions de livres didactiques ont été distribués, ce qui signifie prés de 3 livres par élève de l'enseignement public (Ferreira, 1995). 
Mais, comme l'affirme Ferreira (1995), malgré quelques innovations intéressantes comme par exemple la possibilité donnée aux enseignants de choisir parmi une liste de manuels scolaires fournie par les éditeurs début de chaque année scolaire, nous pouvons dire que ce programme supposé améliorer la qualité de l'enseignement public de base a servi en réalité à financer les intérêts et les nécessités de quelques éditeurs privés.

En 1987, 5 grands éditeurs se sont partagés 85 \% d'un marché de plus de 50 millions de livres laissant à peine $15 \%$ pour une quarantaine de petits éditeurs.

Les enjeux financiers sont énormes ...La plupart des éditeurs sont situés à Sao Paulo, mais il y a quand même un Projet Nord-Est de la Banque Mondiale.

Le marché du livre didactique des écoles publiques est l'enjeu les intérêts privés monopolistiques l'emportent largement sur la nécessité d'améliorer l'enseignement public et ses ressources pédagogiques.

Ferreira (1995) estime que la nouvelle république brésilienne peut être considérée comme une période de transition à l'intérieur de la réforme conservatrice. Sa trajectoire correspond à une conciliation par le haut avec un élargissement du circuit élitiste et une libération du contrôle du pouvoir sans que les traditionnels exclus ne soient véritablement intégrés comme acteurs légitimes dans les décisions. Il s'agit d "un changement de tutelle" avec la participation à l'accès aux biens de consommation, mais une exclusion dans la décision et le contrôle politique ".

L'Etat a reconnu (sur le plan du discours) que le plein accès à la citoyenneté n'est pas possible sans résoudre le problème d'accès, de permanence et d'aboutissement de la scolarité des plus exclus.

Ensuite, nous devons examiner les conditions nécessaires pour s'attaquer aux inégalités éducatives structurelles. Ceci n'est possible qu'en rompant avec la logique des réseaux à plusieurs vitesses. Autrement dit comment augmenter la vitesse du réseau public pour la rendre comparable à celle du réseau privé.

La question est tout d'abord financière dans la mesure ou il existe une insuffisance en matière de dépenses pour l'éducation publique. Il est certain que proposer l'augmentation des dépenses pour l'éducation publique peut paraitre provocateur pour certains économistes dans la mesure où la période actuelle caractérisée par les restrictions budgétaires comme religion. Mais quand on observe que le gouvernement fédéral a dépensé milliards de \$ pour soutenir des banques privées ou la parité de la monnaie nationale avec le dollar, on s'étonnera qu'il ne trouve pas les financements nécessaires pour l'éducation publique.

De surcroît, il existe de multiples financements bilatéraux ou multilatéraux pour l'éducation de base comme ceux de la Banque Mondiale 
qui sont malheureusement orienté vers les réalisations matérielles voyantes (Akkari, Article Australie).

De même, l'arrêt du financement public des entreprises privées est nécessaire. Une réflexion nationale sur les manuels scolaires est urgente. En effet, l'argent public gagné par les entrepreneurs privés du secteur est une situation provocante.

Une nécessaire mobilisation du corps enseignant et des spécialistes des sciences de l'éducation est également urgente. Cette remobilisation passe par la nécessité de dénoncer le mythe du marché libre des services éducatifs. En effet, ce marché n'est que l'une des facettes de la distribution injuste de la rente. Moins de $15 \%$ des brésiliens contrôlent $90 \%$ de la richesse crée dans le pays (Da Silva, 1998).

Dans la mesure où chaque réseau s'adresse à des consommateurs différents, le système éducatif brésilien n'est ni un marché libre, ni concurrentiel.

Les salaires des enseignants de l'éducation publique est également un aspect important de la remobilisation en faveur de l'éducation publique.

Les enseignants du réseau public peuvent se mobiliser professionnellement, mais aussi sur le plan personnel, pour remettre leurs enfants dans les écoles publiques, le rapport de force en faveur de l'éducation publique peut alors changer durablement.

Le mouvement des écoles communautaires a émergé au Nord et au Nord-Est du Brésil durant les années 70 à cause de l'insuffisance du secteur public et l'inaccessibilité de l'éducation privée pour les pauvres. Au début des années 90, les associations des éducateurs des écoles communautaires ont émergé comme une importante force politique dans le mouvement populaire brésilien. Appuyé par diverses O.N.G notamment le centre d'éducation populaire, ce mouvement s'est mobilisé pour obtenir la reconnaissance légale et le support financier pour les écoles communautaires. En 1998, un amendement constitutionnel reconnaissait l'existence légale de ces écoles.

L'expérience éducative du mouvement des sans terre (MST) est également intéressante. Des milliers d'élèves sont actuellement scolarisé dans les écoles gérées par le mouvement. Une première rencontre nationale des éducateurs du MST a été organisé récemment à Brasilia.

Les expériences des municipalités de Gauche à Porto Alegre ou à Brasilia peuvent fournir des motifs de mobilisation.

Sur le plan du débat pédagogique, il est nécessaire de déplacer les discussions de la psychologie de l'éducation, dominée par Vygotsky et Piaget vers la sociologie de l'éducation et la critique sociale du néolibéralisme. Non pas que la psychopédagogie ne soit pas capitale dans la compréhension des enjeux éducatifs, mais parce qu'elle n'inclut généralement pas les enjeux politiques et contextuels du système éducatif. Or réfléchir sur une réforme du curriculum [reformas curiculares] dans un Etat brésilien ne peut pas se faire à 
l'heure actuelle sans une analyse approfondie et critique des rapports entre éducation publique et éducation privée. Aucune réforme ne peut fonctionner dans un système à plusieurs vitesses structuré en fonction de l'intérêt exclusif des classes dominantes.

Malgré le retentissement international de son œuvre, Freire ne s'est intéressé que vers la fin de sa carrière à l'éducation scolaire de base.

\section{Conclusion}

Dans cet article, nous avons tenté de démontrer que le système éducatif actuel brésilien n'est pas une photocopie du modèle occidental ou européen puisqu'il diverge sur bien des aspects par rapport à ce dernier. La monté en puissance du secteur privé (primaire et secondaire) seul habiliter à reproduire les élites se retrouvent également dans d'autres régions du Tiers-Monde, il serait dont utile de réfléchir sur la possibilité d'une troisième voie que nous pouvons dénommer en empruntant l'expression de P. Freire, Ecole publique populaire.

\section{Nota bibliográfica}

1 Ancien sociologue marxiste, le président Cardoso a choisit de s'allier pour gouverner aux franges les plus rétrogrades de la droite brésilienne.

\section{Références}

AZEVEDO, F. et al. (1932). O manifesto dos pioneiros da educação nova. Sao Paulo : Cia. Ed. Nacional.

CAREIL, Y. (1998). De l'école publique à l'école libérale. Sociologie d'un changement. Rennes: Presses Universitaires de Rennes.

SILVA, A.R. Da (1998). A importância da educação publica para o ensino fundamental das classes trabalhadoras. Cadernos de apoio ao ensino UEM,4, 5-42.

CASTRO, C. De Moura (19997). Ensino Privado ou público : Eis a (falsa) questao. Ensaio, 5 (17), 423-452.

PESTANA, Gomes de Sá et al. (1999). Saeb 97: primeros resultados. Brasília: Instituto Nacional de Estudos e Pesquisas Educacionais.

GUIMARAES, M.H. (1998). Avaliação do sitema educacional brasileiro. Tendências e perspectivas. Ensaio, 6(20), 303-364. 
HUTNACHER, W. (1900).

KOZOL, J. (1900). Salvage inequalities.

LIBANEO, J. C. (1985). Democratização da escola pública. A pedagogia crítico-social dos conteudos $(14$ ed.). São Paulo: Edições Loyola.

LIMA, M. (1999, July). Lugar marcado. Projeto de lei propõe cotas nas faculdades do governo para alunos de rede pùblica. Veja, pp 46-47.

MESQUIDA, P. (1994). Hegemonia Norte-Americana e Educacao Protestante no Brasil. Juiz de Fora. São Bernardo do Campo: Editora da UFJF / EDITEO.

SABOIA, J. (1996). Trabalho Infanto - Juvenil no Brasil dos Anos 90. Rio de Janeiro: UNICEF.

SAVIANI, D. (1992). Pedagogia Histórico-crítica. Primeiras aproximações. São Paulo: CORTEZ.

SAVIANI, D. (1996). Escola e Democracia. São Paulo: CORTEZ.

TRAumANn, T. (1999, August) O Paradoxo de ACM. Veja, pp 46-48.

WATKINS, K. (1999). Education now: Break the cycle of Poverety. London: OXFAM

World Bank (1989). Issues in Brazilian secondary education (report $n$. 7723BR, Latin American and Carabbean Regional Office

ZIBAS, D. (1997). Escola pública versus Escola privada: O fim da história? Cadernos de Pesquisa, 100, 57-7.

e-mail: peri@rla01.pucpr.br 